\title{
Trends in stunting and overweight in Peruvian pre-schoolers from 1991 to 2011 : findings from the Demographic and Health Surveys
}

\author{
Helga Bjørnøy Urke ${ }^{1, *}$, Maurice B Mittelmark ${ }^{1}$ and Martín Valdivia ${ }^{2}$ \\ 'Department of Health Promotion and Development, University of Bergen, PB 7807, NO-5020 Bergen, Norway: \\ ${ }^{2}$ Grupo de Análisis para el Desarrollo (GRADE), Lima, Peru
}

Submitted 12 April 2013: Final revision received 6 December 2013: Accepted 5 February 2014: First published online 14 March 2014

\begin{abstract}
Objective: To examine trends in stunting and overweight in Peruvian children, using 2006 WHO Multicentre Growth Reference Study criteria.

Design: Trend analyses using nationally representative cross-sectional surveys from Demographic and Health Surveys (1991-2011). We performed logistic regression analyses of stunting and overweight trends in sociodemographic groups (sex, age, urban-rural residence, region, maternal education and household wealth), adjusted for sampling design effects (strata, clusters and sampling weights).

Setting: Peru.

Subjects: Children aged 0-59 months surveyed in 1991-92 ( $n$ 7999), 1996 ( $n$ 14877), 2000 ( $n$ 11754), 2007-08 ( $n$ 8232) and 2011 ( $n$ 8186).

Results: Child stunting declined $(F(1,5149)=174 \cdot 8, P \leq 0 \cdot 00)$ and child overweight was stable in the period 1991-2011 $(F(1,5147)=0 \cdot 4, P \leq 0 \cdot 54)$. Over the study period, levels of stunting were highest in rural compared with urban areas, the Andean and Amazon regions compared with the Coast, among children of low-educated mothers and among children living in households in the poorest wealth quintile. The trend in overweight rose among males in coastal areas $(F(1,2250)=4 \cdot 779, P \leq 0 \cdot 029)$ and among males in the richest wealth quintile $(F(1,1730)=5 \cdot 458, P \leq 0 \cdot 020)$.

Conclusions: The 2011 levels of stunting and overweight were eight times and three and a half times higher, respectively, than the expected levels from the 2006 WHO growth standards. The trend over the study period in stunting declined in most sociodemographic subgroups. The trend in overweight was stable in most sociodemographic subgroups.
\end{abstract}

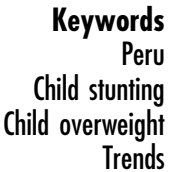

The transition to Western lifestyles in low- and middleincome countries has resulted in the consumption of more energy-dense foods and obesity, while the problem of undernutrition persists ${ }^{(1,2)}$. This phenomenon is characterized as the nutritional dual burden ${ }^{(1)}$. Latin America is no exception to this emerging manifestation of malnutrition $^{(1,3-6)}$. The dual burden may be manifest at various levels; the population level, the household level and the individual level ${ }^{(1)}$. The present paper focuses on the dual burden at the population level.

This is relevant to Peru, which is undergoing transitions both economically and demographically. The economy grew by $7 \%$ in 2011, ranking fourth in the region and above large economies like Mexico, Chile and Brazil ${ }^{(7)}$. Demographically, the population growth is at $1 \cdot 1 \%$ annually, the urban growth is at $1.6 \%$, whereas the rural population is decreasing ${ }^{(8)}$.

Under- and overnutrition are significant public health problems having origins in early childhood. Infant and child stunting are associated with cognitive impairment ${ }^{(9)}$, physical disease and mortality in childhood ${ }^{(10)}$. Stunting in childhood is in turn associated with adult stunting, lower educational attainment and income, and low offspring birth weight ${ }^{(11)}$. Overweight and obese children are at heightened risk of a range of physical health problems ${ }^{(12,13)}$ and infant and childhood overweight increases the risk of subsequent overweight in later childhood, adolescence and adulthood ${ }^{(14)}$. Overweight in adult age is associated with increased risk of health problems like diabetes and CVD and subsequent death ${ }^{(13)}$. From a public health 
perspective, this carry-over nutritional and health effect from infancy and childhood to adulthood points to the urgency of ensuring priority to healthy child nutrition.

While infant and child nutrition is an issue addressed by the research community, there are important limitations in the published analyses. Studies are mostly based on single cross-sectional analyses ${ }^{(15-17)}$. Long-term trends are difficult to ascertain due to the incomparability of data from different time periods and methodological variation. There is a need to establish reliable and valid trend estimates to inform public policy makers about the degree of progress, or lack thereof, in combating malnutrition. Further, very few studies have examined trends covering both stunting and overweight, and those that have tend to examine national and regional trends including several countries, without stratifying by important variables within countries ${ }^{(3,6)}$. As an important example, WHO recommends stratifying anthropometric data by sex and age groups, because children face critical periods in growth development ${ }^{(18)}$. Furthermore, macro-analyses at national level only may mask important variation in child malnutrition patterns at urban-rural, provincial, maternal education and household wealth levels ${ }^{(19)}$.

Moreover and of substantial importance, the research literature is inconsistent in the way malnutrition is operationally defined. Most studies in the literature used now obsolete child growth standards ${ }^{(6,20,21)}$ or used a variety of incomparable cut-off points to define malnutrition $^{(1,12,22,23)}$. The currently recommended WHO child growth standards ${ }^{(24)}$ give higher estimates for both stunting and overweight compared with the earlier standards of the US National Center for Health Statistics ${ }^{(25)}$ and the US Centers for Disease Control and Prevention ${ }^{(26)}$, and this poses serious problems concerning comparison of studies using the various standards. To enable valid comparisons across years, studies are needed that apply the new WHO growth standards to data that pre-date the new standards, as well as newer data. To capture information on subgroup differences within countries, analyses are needed that are stratified by child age, sex and other key sociodemographic variables.

The present study aimed to examine 20-year trends (1991-2011) in stunting and overweight in children aged 0-59 months in Peru, using a study design intended to overcome the limitations described above. The following research question was addressed: what are the nationallevel sex-specific trends in child stunting and overweight in Peru and in subgroups defined by (i) age, (ii) geographic region, (iii) urban/rural split, (iv) maternal education level and (v) household wealth?

\section{Methods}

\section{Study design and sample}

The study used cross-sectional data from Demographic and Health Surveys (DHS) in Peru collected at five time points: 1991-92, 1996, 2000, 2007-08 and 2011. The sampling frame for each DHS survey consists of households selected in two stages to be nationally representative ${ }^{(27)}$. The first stage is strata (domains) defined by regional and urban-rural characteristics. Within strata, clusters (census districts) are selected at random. Within clusters, households are selected systematically and all residents are enumerated. One woman from each household aged 15-49 years is selected to complete a detailed questionnaire. Her youngest living child is selected for anthropometry and questions about health ${ }^{(28)}$. The woman response rates were $92.6 \%$ in $1991-92,92 \cdot 7 \%$ in $1996,94 \cdot 6 \%$ in $2000,97 \cdot 7 \%$ in 2007-08 and $97 \cdot 7 \%$ in $2011^{(29-33)}$. The weighted samples sizes of children aged 0-59 months in the present study were as follows: $n 7999$ in 1991-92, $n 14877$ in 1996, $n 11754$ in 2000, $n 8232$ in 2007-08 and $n 8186$ in 2011.

\section{Antbropometric measurements}

The study applied the currently recommended WHO Child Growth Standards for international use ${ }^{(24,26)}$. $Z$-scores and the prevalences of stunting and overweight were computed using a syntax file provided by $\mathrm{WHO}^{(34)}$. Stunting is defined as height-for-age less than $-2 \mathrm{SD}$ below the median of the reference population (heightfor-age $Z$-score (HAZ) $<-2$ ), and overweight is defined as weight-for-height more than $+2 \mathrm{SD}$ above the median of the reference population (weight-for-height $Z$-score $(\mathrm{WHZ})>+2$ ). Extreme values beyond $+/-6$ SD were excluded according to recommendations by $\mathrm{WHO}^{(35)}$. Supine length was measured for children below 24 months and standing height was measured for children over 24 months. Details on anthropometric measurement in DHS can be found elsewhere ${ }^{(36)}$. Personnel with responsibility for anthropometric measures data collection received special training by professionals before each data collection. Data were later verified for consistency ${ }^{(29-33)}$.

\section{Sociodemographic variables}

Sociodemographic variables used for stratification were sex, age in months $(0-5,6-11,12-23,24-35,36-47$, 48-59), urban-rural residence, geographic region, maternal education and household wealth. Data on the latter four variables were obtained through household questionnaires ${ }^{(29-33)}$. Wealth was assessed using a standard composite Wealth Index based on household material goods and housing quality ${ }^{(37)}$. Child age was documented by asking the mother the day, month and year the child was born, as well as how many years old the child was at time of interview ${ }^{(29-33)}$.

\section{Etbical approval}

Data were collected with informed consent and anonymity of respondents was assured ${ }^{(29-32)}$. The questionnaires and protocols were reviewed and approved by the Macro 
Institutional Review Board and the Peruvian National Institute for Statistics (INEI) Ethics Board.

\section{Data analysis}

Data analyses were performed using the statistical software package IBM SPSS Statistics $19 \cdot 0$. Data sets from all five survey rounds were combined through the 'merge' function in IBM SPSS Statistics $19 \cdot 0$. Sample weights were used to account for unequal probability of sampling and for non-response. Multilevel analyses accounted for sampling strata and clusters. Logistic regression analyses examined trends over time within subgroups and between subgroups. The regression was performed of stunting and overweight prevalence $v$. time represented by the five data collection points (Tables 1-6). The Wald $F$ statistic was used to test the significance of trends $(P<0 \cdot 05)$. For comparison purposes, the national levels of stunting/overweight are included in the first row of Tables 1-4.

\section{Results}

\section{Stunting}

Across all survey years, 4630 of 56168 (8.2\%) of children who were eligible for anthropometry measurement were excluded from the present study due to measurement problems including missing measurements and out-ofrange values for growth $Z$-scores $(<-6 \cdot 00$ and $>+6 \cdot 00)$. As shown in Tables 1 and 2, stunting nationally decreased significantly from $37 \cdot 4 \%$ in $1991-92$ to $19 \cdot 3 \%$ in $2011(F(1,5149)=174 \cdot 8, P \leq 0 \cdot 00)$. Analyses for sociodemographic subgroups largely showed similar, statistically significant declining trends for both sexes. The results reported below concentrate on the exceptions to the general declining trend and on differences in the steepness of declines in subgroups. The only marked exception to the overall declining trend was observed for males and females in the age groups $0-5$ and 6-11 months, in which stunting prevalence remained stable over the period (Tables 1 and 2).

Also, as shown in Table 6, there were some instances in which the steepness of declines varied significantly. Stunting in urban areas declined more steeply over the study period compared with rural areas $(F(1,5149)=16 \cdot 8$, $P \leq 0 \cdot 00)$. For geographic region, in comparison with the Andes, the decline in stunting was significantly steeper in the Coastal region $(F(1,4094)=15 \cdot 2, P \leq 0 \cdot 00)$ and in the Amazon region $(F(1,2954)=35 \cdot 6, P \leq 0 \cdot 00)$. With regard to educational attainment of mothers, the decline in stunting was significantly steeper among children having mothers in the highest education group compared with those in the primary education group $(F(1,4925)=7 \cdot 9$, $P \leq 0 \cdot 01)$. The comparison of the steepness of declines was also undertaken between age groups and Wealth Index quintiles, with no significant differences revealed.

\section{Overweight}

For overweight across all survey years, the (unadjusted) number excluded due to anthropometry measurement problems was 4895 of the total sample of 56168 (8.7\%). The national trend in overweight was stable across the study period $(F(1,5147)=0 \cdot 4, P \leq 0 \cdot 5)$ and this was also observed in the sex-specific analyses (Tables 3 and 4 ). Subgroup analyses, however, revealed exceptions of both worsening and improving trends. The instances of statistically significant worsening trends were for Coastal males $(F(1,2255)=4 \cdot 8, P \leq 0 \cdot 03)$ and highest wealth quintile males $(F(1,1730)=5 \cdot 5, P \leq 0 \cdot 02)$.

Statistically significant declining trends in overweight were observed in the subgroups of females aged 24-35 months $(F(1,4073)=4 \cdot 8, P \leq 0 \cdot 03)$, rural males $(F(1,1943)=8 \cdot 7, P \leq 0 \cdot 00)$, rural females $(F(1,1943)=6 \cdot 0$, $P \leq 0 \cdot 02)$, Amazon region full sample $(F(1,1096)=35 \cdot 3$, $P \leq 0 \cdot 00)$, Andean region full sample $(F(1,1890)=12 \cdot 7$, $P \leq 0 \cdot 00)$, incomplete secondary education full sample $(F(1,3289)=24 \cdot 7, \quad P \leq 0 \cdot 00)$ and incomplete primary education full sample $(F(1,2959)=12 \cdot 5, P \leq 0 \cdot 00)$. As shown in Table 5 , there was also a significantly steeper decline in the Amazon region compared with the Andes $(F(1,2953)=8 \cdot 6, P \leq 0 \cdot 00){ }^{*}$

\section{Discussion}

Over the 20-year period examined, the present study found a statistically significant decrease in overall child stunting and no overall change in the level of child overweight in Peru. This is the first publication of child malnutrition trends over such an extended period using the same operational definitions of stunting and overweight for all data points. While the overall findings summarized above were reflected to a large degree in analyses that examined trends by sociodemographic subgroups, there were important exceptions (for example, stunting was stable in the two youngest age groups, but decreased in the three eldest). These results suggest that while national-level data are appropriate for international comparative studies, stratified analyses are called for when the public health profile within a particular country is the focus.

Indeed, the observation of some encouraging trends should not cause complacency. Seen from the standpoint of health equity, the national estimate of $19 \cdot 3 \%$ child stunting in 2011 is almost eight times the level of stunting in the WHO reference group. Examining the subgroups with the highest stunting estimates in Peru in 2011 children of mothers with no education - males are at twenty times and females are at twenty-three times the

\footnotetext{
* As mentioned in the introduction, the current paper focuses on the dual burden of stunting and overweight at the population level. However, because there is growing interest in the dual burden at the individual level, we have estimated its prevalence with the present data at the aggregate level: $2 \cdot 9 \%$ in $1991-92,2 \cdot 4 \%$ in $1996,3 \cdot 0 \%$ in $2000,2 \cdot 1 \%$ in 2007-08 and $0.6 \%$ in 2011.
} 
Table 1 Prevalence estimates for stunting (height-for-age $<-2$ so below the median of the reference population*) in males (aged 0-59 months) and test for trend over time by sociodemographic factors. Peru Demographic and Health Surveys, 1991-2011

\begin{tabular}{|c|c|c|c|c|c|c|c|c|c|c|c|c|c|c|c|c|c|c|}
\hline \multirow[b]{2}{*}{ Variable } & \multicolumn{3}{|c|}{ 1991-92 } & \multicolumn{3}{|c|}{1996} & \multicolumn{3}{|c|}{2000} & \multicolumn{3}{|c|}{$2007-08$} & \multicolumn{3}{|c|}{2011} & \multirow[b]{2}{*}{ Wald $(F)$} & \multirow[b]{2}{*}{$P+$} & \multirow[b]{2}{*}{ df } \\
\hline & $n$ & $\%$ & $95 \% \mathrm{Cl}$ & $n$ & $\%$ & $95 \% \mathrm{Cl}$ & $n$ & $\%$ & $95 \% \mathrm{Cl}$ & $n$ & $\%$ & $95 \% \mathrm{Cl}$ & $n$ & $\%$ & $95 \% \mathrm{Cl}$ & & & \\
\hline Total both sexes & 7138 & $37 \cdot 4$ & $35 \cdot 4,39 \cdot 4$ & 13611 & $31 \cdot 8$ & $30 \cdot 3,33 \cdot 3$ & 10585 & $31 \cdot 4$ & $29 \cdot 8,33 \cdot 0$ & 7403 & $27 \cdot 9$ & $26 \cdot 0,29 \cdot 9$ & 8074 & $19 \cdot 3$ & $17 \cdot 8,20 \cdot 9$ & $174 \cdot 8$ & $\leq 0.00$ & 1,5149 \\
\hline & \multicolumn{8}{|c|}{ Age (months) } & $29 \cdot 8,33 \cdot 5$ & 3713 & $29 \cdot 3$ & $27 \cdot 0,31 \cdot 7$ & 4118 & $19 \cdot 8$ & $17 \cdot 9,21 \cdot 7$ & $105 \cdot 7$ & $\leq 0.00$ & 1,5149 \\
\hline $0-5$ & 519 & $14 \cdot 1$ & $11 \cdot 1,17 \cdot 7$ & 895 & $14 \cdot 0$ & $11 \cdot 4,17 \cdot 1$ & 596 & $14 \cdot 5$ & $11 \cdot 4,18 \cdot 3$ & 444 & $15 \cdot 6$ & $11 \cdot 9,20 \cdot 2$ & 453 & $13 \cdot 3$ & $10 \cdot 2,17 \cdot 2$ & $0 \cdot 0$ & $\leq 0.96$ & 1,2868 \\
\hline $6-11$ & 483 & $25 \cdot 8$ & $22 \cdot 0,30 \cdot 0$ & 914 & $21 \cdot 0$ & $18 \cdot 0,24 \cdot 3$ & 619 & $20 \cdot 5$ & $17 \cdot 2,24 \cdot 3$ & 434 & $26 \cdot 6$ & $21 \cdot 0,33 \cdot 1$ & 455 & $20 \cdot 5$ & $16 \cdot 4,25 \cdot 4$ & 0.3 & $\leq 0.61$ & 1,2927 \\
\hline $12-23$ & 840 & $41 \cdot 8$ & $37 \cdot 9,45 \cdot 8$ & 1675 & $40 \cdot 6$ & $37 \cdot 4,43 \cdot 8$ & 1332 & $36 \cdot 8$ & $33 \cdot 5,40 \cdot 2$ & 808 & $36 \cdot 0$ & $31 \cdot 6,40 \cdot 6$ & 922 & $25 \cdot 4$ & $21 \cdot 9,29 \cdot 2$ & $37 \cdot 4$ & $\leq 0.00$ & 1,4039 \\
\hline $24-35$ & 750 & $48 \cdot 1$ & $43 \cdot 9,52 \cdot 3$ & 1341 & $39 \cdot 8$ & $36 \cdot 4,43 \cdot 3$ & 1043 & $38 \cdot 5$ & $34 \cdot 9,42 \cdot 1$ & 764 & 34.5 & $29 \cdot 4,39 \cdot 9$ & 947 & $20 \cdot 4$ & $17 \cdot 3,23 \cdot 9$ & $85 \cdot 6$ & $\leq 0.00$ & 1,4082 \\
\hline $36-47$ & 611 & $40 \cdot 1$ & $35 \cdot 9,44 \cdot 4$ & 1112 & $34 \cdot 2$ & $31 \cdot 0,37 \cdot 5$ & 960 & $36 \cdot 0$ & $32 \cdot 2,40 \cdot 0$ & 708 & $29 \cdot 3$ & $25 \cdot 1,34 \cdot 0$ & 726 & $19 \cdot 4$ & $16 \cdot 1,23 \cdot 9$ & $48 \cdot 8$ & $\leq 0.00$ & 1,4148 \\
\hline $48-59$ & 494 & $38 \cdot 9$ & $34 \cdot 2,43 \cdot 8$ & 952 & $30 \cdot 1$ & $26 \cdot 7,33 \cdot 7$ & 866 & $30 \cdot 1$ & $26 \cdot 5,33 \cdot 9$ & 581 & $25 \cdot 5$ & $21 \cdot 3,30 \cdot 3$ & 616 & $14 \cdot 9$ & $11 \cdot 8,18 \cdot 6$ & $54 \cdot 3$ & $\leq 0.00$ & 1,4009 \\
\hline \multicolumn{19}{|l|}{ Residence } \\
\hline Urban & 2260 & $25 \cdot 8$ & $23 \cdot 6,28 \cdot 2$ & 4139 & $22 \cdot 3$ & $20 \cdot 4,24 \cdot 4$ & 2937 & $19 \cdot 1$ & $17 \cdot 1,21 \cdot 2$ & 2240 & $16 \cdot 7$ & $14 \cdot 4,19 \cdot 2$ & 2733 & $10 \cdot 9$ & $9 \cdot 2,12 \cdot 9$ & $100 \cdot 6$ & $\leq 0.00$ & 1,3334 \\
\hline Rural & 1437 & $53 \cdot 1$ & $49 \cdot 7,56 \cdot 4$ & 2750 & $46 \cdot 3$ & $43 \cdot 8,48 \cdot 8$ & 2480 & $46 \cdot 4$ & $44 \cdot 0,48 \cdot 9$ & 1499 & $48 \cdot 1$ & $44 \cdot 7,51 \cdot 6$ & 1385 & $37 \cdot 2$ & $34 \cdot 1,40 \cdot 4$ & $27 \cdot 2$ & $\leq 0.00$ & 1,1944 \\
\hline \multicolumn{19}{|l|}{ Geographic region } \\
\hline Coast & 1759 & $22 \cdot 9$ & $20 \cdot 5,25 \cdot 5$ & 3119 & $18 \cdot 7$ & $16 \cdot 6,20 \cdot 9$ & 2346 & $16 \cdot 7$ & $14 \cdot 5,19 \cdot 2$ & 1625 & $15 \cdot 3$ & $12 \cdot 7,18 \cdot 4$ & 1646 & $8 \cdot 3$ & $6 \cdot 4,10 \cdot 7$ & $63 \cdot 0$ & $\leq 0 \cdot 00$ & 1,2256 \\
\hline Andes & 501 & $36 \cdot 2$ & $31 \cdot 1,41 \cdot 6$ & 2721 & $44 \cdot 2$ & $41 \cdot 8,46 \cdot 7$ & 2586 & $44 \cdot 1$ & $41 \cdot 7,46 \cdot 5$ & 1621 & $43 \cdot 1$ & $39 \cdot 0,47 \cdot 3$ & 1166 & $29 \cdot 7$ & $26 \cdot 4,33 \cdot 2$ & $14 \cdot 7$ & $\leq 0.00$ & 1,1890 \\
\hline Amazon basin & 1437 & $53 \cdot 1$ & $49 \cdot 7,56 \cdot 4$ & 1049 & $39 \cdot 3$ & $35 \cdot 9,42 \cdot 8$ & 484 & $37 \cdot 0$ & $32 \cdot 6,41 \cdot 6$ & 4932 & $29 \cdot 8$ & $25 \cdot 7,34 \cdot 4$ & 500 & $26 \cdot 5$ & $22 \cdot 9,30 \cdot 5$ & $114 \cdot 5$ & $\leq 0.00$ & 1,1097 \\
\hline \multicolumn{19}{|c|}{ Maternal education } \\
\hline Complete secondary/higher & 1161 & $16 \cdot 7$ & $14 \cdot 4,19 \cdot 4$ & 2188 & $13 \cdot 9$ & $12 \cdot 1,15 \cdot 9$ & 1969 & $13 \cdot 3$ & $11 \cdot 4,15 \cdot 4$ & 1662 & $13 \cdot 8$ & $11 \cdot 6,16 \cdot 3$ & 2047 & $7 \cdot 7$ & $6 \cdot 3,9 \cdot 4$ & $31 \cdot 4$ & $\leq 0.00$ & 1,4126 \\
\hline Incomplete secondary & 660 & $28 \cdot 1$ & $24 \cdot 4,32 \cdot 2$ & 1251 & $27 \cdot 8$ & $24 \cdot 9,30 \cdot 9$ & 848 & $25 \cdot 6$ & $22 \cdot 4,29 \cdot 1$ & 630 & $29 \cdot 0$ & $24 \cdot 7,33 \cdot 7$ & 688 & $20 \cdot 5$ & $16 \cdot 9,24 \cdot 7$ & $5 \cdot 5$ & $\leq 0.02$ & 1,3289 \\
\hline Complete primary & 742 & $44 \cdot 6$ & $41 \cdot 1,48 \cdot 2$ & 1224 & $37 \cdot 2$ & $34 \cdot 0,40 \cdot 6$ & 906 & $40 \cdot 7$ & $37 \cdot 1,44 \cdot 4$ & 538 & $38 \cdot 8$ & $33 \cdot 6,44 \cdot 3$ & 544 & $33 \cdot 3$ & $29 \cdot 1,37 \cdot 8$ & $7 \cdot 9$ & $\leq 0.01$ & 1,2915 \\
\hline Incomplete primary & 767 & $52 \cdot 4$ & $48 \cdot 6,56 \cdot 3$ & 1575 & $47 \cdot 9$ & $44 \cdot 7,51 \cdot 0$ & 1273 & $49 \cdot 1$ & $45 \cdot 9,52 \cdot 4$ & 746 & $49 \cdot 6$ & $45 \cdot 1,54 \cdot 1$ & 708 & $37 \cdot 8$ & $33 \cdot 3,42 \cdot 5$ & $13 \cdot 4$ & $\leq 0.00$ & 1,296 \\
\hline No education & 368 & 63.5 & $57 \cdot 9,68 \cdot 9$ & 651 & $51 \cdot 8$ & $47 \cdot 1,56 \cdot 5$ & 421 & $56 \cdot 6$ & $51 \cdot 3,61 \cdot 7$ & 163 & $64 \cdot 1$ & $56 \cdot 1,71 \cdot 4$ & 132 & $50 \cdot 8$ & $41 \cdot 4,60 \cdot 3$ & 0.7 & $\leq 0.39$ & 1,1271 \\
\hline \multicolumn{19}{|l|}{ Wealth quintile } \\
\hline Richest & 344 & $7 \cdot 8$ & $5 \cdot 0,11 \cdot 9$ & 728 & $10 \cdot 5$ & $7 \cdot 9,14 \cdot 0$ & 655 & $8 \cdot 0$ & $5 \cdot 6,11 \cdot 5$ & 713 & $7 \cdot 7$ & $4 \cdot 7,12 \cdot 5$ & 340 & $3 \cdot 5$ & $1 \cdot 8,6 \cdot 6$ & $6 \cdot 7$ & $\leq 0 \cdot 01$ & 1,1734 \\
\hline Richer & 579 & $14 \cdot 5$ & $11 \cdot 3,18 \cdot 4$ & 1155 & $17 \cdot 4$ & $14 \cdot 4,21 \cdot 0$ & 868 & $12 \cdot 2$ & $9 \cdot 6,15 \cdot 4$ & 648 & $13 \cdot 7$ & $10 \cdot 2,18 \cdot 0$ & 611 & $7 \cdot 9$ & $5 \cdot 3,11 \cdot 7$ & $11 \cdot 6$ & $\leq 0.00$ & 1,2414 \\
\hline Middle & 732 & $29 \cdot 5$ & $25 \cdot 7,33 \cdot 6$ & 1452 & $23 \cdot 8$ & $20 \cdot 8,27 \cdot 1$ & 1066 & $22 \cdot 8$ & $19 \cdot 8,26 \cdot 1$ & 910 & $26 \cdot 8$ & $23 \cdot 1,30 \cdot 9$ & 952 & $10 \cdot 9$ & $8 \cdot 4,14 \cdot 0$ & $34 \cdot 0$ & $\leq 0.00$ & 1,2952 \\
\hline Poorer & 959 & $42 \cdot 4$ & $38 \cdot 9,45 \cdot 9$ & 1684 & $37 \cdot 6$ & $34 \cdot 8,40 \cdot 5$ & 1329 & $38 \cdot 2$ & $35 \cdot 1,41 \cdot 4$ & 1017 & $44 \cdot 3$ & $40 \cdot 3,48 \cdot 3$ & 1129 & $22 \cdot 6$ & $19 \cdot 6,25 \cdot 9$ & $26 \cdot 9$ & $\leq 0.00$ & 1,3020 \\
\hline Poorest & 1082 & $56 \cdot 7$ & $53 \cdot 0,60 \cdot 3$ & 1870 & $50 \cdot 3$ & $47 \cdot 2,53 \cdot 5$ & 1499 & $53 \cdot \overline{5}$ & $50 \cdot 7,56 \cdot 3$ & 452 & $57 \cdot 0$ & $51 \cdot 0,62 \cdot 8$ & 1400 & 43.5 & $40 \cdot 1,47 \cdot 0$ & $12 \cdot 9$ & $\leq 0.00$ & 1,2010 \\
\hline
\end{tabular}

*Using the 2006 WHO Child Growth Standards ${ }^{(24)}$

tStatistical significance of trends across years within each subgroup $(P \leq 0.05)$ 
Table 2 Prevalence estimates for stunting (height-for-age $<-2$ sD below the median of the reference population ${ }^{\star}$ ) in females (aged 0-59 months) and test for trend over time by sociodemographic factors. Peru Demographic and Health Surveys, 1991-2011

\begin{tabular}{|c|c|c|c|c|c|c|c|c|c|c|c|c|c|c|c|c|c|c|}
\hline \multirow[b]{2}{*}{ Variable } & \multicolumn{3}{|c|}{ 1991-92 } & \multicolumn{3}{|c|}{1996} & \multicolumn{3}{|c|}{2000} & \multicolumn{3}{|c|}{$2007-08$} & \multicolumn{3}{|c|}{2011} & \multirow[b]{2}{*}{ Wald $(F)$} & \multirow[b]{2}{*}{$P+$} & \multirow[b]{2}{*}{ df } \\
\hline & $n$ & $\%$ & $95 \% \mathrm{Cl}$ & $n$ & $\%$ & $95 \% \mathrm{Cl}$ & $n$ & $\%$ & $95 \% \mathrm{Cl}$ & $n$ & $\%$ & $95 \% \mathrm{Cl}$ & $n$ & $\%$ & $95 \% \mathrm{Cl}$ & & & \\
\hline Total both sexes & 7138 & $37 \cdot 4$ & $35 \cdot 4,39 \cdot 4$ & 13611 & $31 \cdot 8$ & $30 \cdot 3,33 \cdot 3$ & 10585 & $31 \cdot 4$ & $29 \cdot 8,33 \cdot 0$ & 7403 & $27 \cdot 9$ & $26 \cdot 0,29 \cdot 9$ & 8074 & $19 \cdot 3$ & $17 \cdot 8,20 \cdot 9$ & $174 \cdot 8$ & $\leq 0.00$ & 1,5149 \\
\hline Total females & 3441 & $38 \cdot 4$ & $36 \cdot 2,40 \cdot 7$ & 6722 & $31 \cdot 7$ & $29 \cdot 9,33 \cdot 5$ & 5169 & $31 \cdot 1$ & $29 \cdot 2,33 \cdot 1$ & 3664 & $26 \cdot 4$ & $24 \cdot 3,28 \cdot 7$ & 3955 & $18 \cdot 8$ & $17 \cdot 0,20 \cdot 8$ & $155 \cdot 6$ & $\leq 0.00$ & 1,5149 \\
\hline \multicolumn{19}{|l|}{ Age (months) } \\
\hline $6-11$ & 267 & 14.9 & $11 \cdot 0,19 \cdot 9$ & 501 & $16 \cdot 0$ & $12 \cdot 7,19 \cdot 8$ & 388 & $10 \cdot 3$ & $7 \cdot 5,14 \cdot 0$ & 293 & $15 \cdot 1$ & $11 \cdot 0,20 \cdot 2$ & 361 & $14 \cdot 0$ & $10 \cdot 2,19 \cdot 0$ & 0.3 & $\leq 0.61$ & 1,2927 \\
\hline $12--23$ & 509 & $30 \cdot 7$ & $26 \cdot 5,35 \cdot 2$ & 1080 & $28 \cdot 8$ & $25 \cdot 5,32 \cdot 4$ & 834 & $26 \cdot 8$ & $23 \cdot 3,30 \cdot 6$ & 667 & $28 \cdot 3$ & $23 \cdot 9,33 \cdot 2$ & 671 & $21 \cdot 1$ & $17 \cdot 6,25 \cdot 2$ & 8.5 & $\leq 0.00$ & 1,4039 \\
\hline $24-35$ & 633 & $45 \cdot 4$ & $41 \cdot 1,49 \cdot 8$ & 1316 & $34 \cdot 3$ & $31 \cdot 1,37 \cdot 7$ & 1041 & $35 \cdot 9$ & $32 \cdot 4,39 \cdot 6$ & 710 & $26 \cdot 8$ & $22 \cdot 8,31 \cdot 2$ & 822 & $19 \cdot 0$ & $16 \cdot 0,22 \cdot 4$ & $84 \cdot 4$ & $\leq 0.00$ & 1,4082 \\
\hline $36-47$ & 971 & $45 \cdot 9$ & $42 \cdot 3,49 \cdot 5$ & 1653 & 38.5 & $35 \cdot 5,41 \cdot 7$ & 1190 & $39 \cdot 2$ & $35 \cdot 7,42 \cdot 8$ & 846 & $31 \cdot 1$ & $26 \cdot 9,35 \cdot 7$ & 847 & $20 \cdot 9$ & $17 \cdot 8,24 \cdot 3$ & $84 \cdot 9$ & $\leq 0.00$ & 1,4148 \\
\hline $48-59$ & 891 & $46 \cdot 2$ & $42 \cdot 5,49 \cdot 9$ & 1713 & $34 \cdot 4$ & $31 \cdot 6,37 \cdot 3$ & 1344 & $35 \cdot 4$ & $32 \cdot 0,38 \cdot 9$ & 899 & $27 \cdot 6$ & $23 \cdot 6,32 \cdot 0$ & 945 & $19 \cdot 4$ & $16 \cdot 3,22 \cdot 9$ & $90 \cdot 9$ & $\leq 0.00$ & 1,4009 \\
\hline \multicolumn{19}{|l|}{ Residence } \\
\hline Urban & 2109 & $27 \cdot 1$ & $24 \cdot 7,29 \cdot 6$ & 4061 & $19 \cdot 8$ & $18 \cdot 0,21 \cdot 8$ & 2874 & $17 \cdot 3$ & $15 \cdot 4,19 \cdot 5$ & 2174 & $15 \cdot 6$ & $13 \cdot 6,18 \cdot 0$ & 2567 & $9 \cdot 2$ & $7 \cdot 8,10 \cdot 8$ & $135 \cdot 3$ & $\leq 0 \cdot 00$ & 1,3334 \\
\hline Rural & 1332 & $56 \cdot 4$ & $53 \cdot 2,59 \cdot 5$ & 2661 & $49 \cdot 7$ & $46 \cdot 8,52 \cdot 7$ & 2295 & $48 \cdot 4$ & $45 \cdot 8,51 \cdot 0$ & 1490 & $42 \cdot 1$ & $38 \cdot 6,45 \cdot 8$ & 1389 & $36 \cdot 7$ & $33 \cdot 0,40 \cdot 4$ & $63 \cdot 7$ & $\leq 0.00$ & 1,1944 \\
\hline \multicolumn{19}{|l|}{ Geographic region } \\
\hline Coast & 1655 & $24 \cdot 8$ & $22 \cdot 2,27 \cdot 6$ & 3153 & $17 \cdot 3$ & $15 \cdot 2,19 \cdot 7$ & 2370 & $15 \cdot 1$ & $12 \cdot 7,17 \cdot 8$ & 1584 & $15 \cdot 1$ & $12 \cdot 4,18 \cdot 4$ & 1577 & $6 \cdot 8$ & $5 \vee 1,9 \cdot 0$ & $75 \cdot 8$ & $\leq 0.00$ & 1,2256 \\
\hline Andes & 454 & $35 \cdot 2$ & $29 \cdot 8,41 \cdot 1$ & 2617 & $45 \cdot 6$ & $42 \cdot 9,48 \cdot 4$ & 2364 & $45 \cdot 8$ & $43 \cdot 3,48 \cdot 4$ & 1615 & $37 \cdot 0$ & $33 \cdot 4,40 \cdot 7$ & 1158 & $29 \cdot 6$ & $26 \cdot 4,33 \cdot 1$ & $33 \cdot 3$ & $\leq 0.00$ & 1,189 \\
\hline Amazon basin & 1332 & $56 \cdot 4$ & $53 \cdot 2,59 \cdot 5$ & 952 & $40 \cdot 8$ & $37 \cdot 0,44 \cdot 8$ & 435 & $38 \cdot 8$ & $34 \cdot 4,43 \cdot 5$ & 466 & $28 \cdot 2$ & $24 \cdot 0,32 \cdot 9$ & 467 & $23 \cdot 5$ & $19 \cdot 9,27 \cdot 5$ & $161 \cdot 9$ & $\leq 0.00$ & 1,1097 \\
\hline \multicolumn{19}{|l|}{ Maternal education } \\
\hline Complete secondary/higher & 1115 & $16 \cdot 4$ & $14 \cdot 1,19 \cdot 0$ & 2213 & $11 \cdot 5$ & $9 \cdot 7,13 \cdot 6$ & 1889 & $12 \cdot 8$ & $11 \cdot 0,14 \cdot 8$ & 1605 & $11 \cdot 8$ & $9 \cdot 8,14 \cdot 2$ & 1958 & $7 \cdot 7$ & $6 \cdot 3,9 \cdot 4$ & $24 \cdot 6$ & $\leq 0 \cdot 00$ & 1,4126 \\
\hline Incomplete secondary & 584 & $32 \cdot 9$ & $29 \cdot 1,36 \cdot 9$ & 1195 & $24 \cdot 9$ & $22 \cdot 0,28 \cdot 0$ & 861 & $27 \cdot 5$ & $24 \cdot 0,31 \cdot 2$ & 593 & $24 \cdot 5$ & $20 \cdot 2,29 \cdot 4$ & 701 & $15 \cdot 7$ & $12 \cdot 7,19 \cdot 2$ & $28 \cdot 1$ & $\leq 0 \cdot 00$ & 1,3289 \\
\hline Complete primary & 655 & $41 \cdot 4$ & $37 \cdot 4,45 \cdot 5$ & 1157 & $35 \cdot 7$ & $32 \cdot 0,39 \cdot 5$ & 881 & $38 \cdot 1$ & $34 \cdot 3,42 \cdot 1$ & 492 & $35 \cdot 5$ & $31 \cdot 1,40 \cdot 2$ & 500 & $32 \cdot 2$ & $27 \cdot 6,37 \cdot 2$ & $5 \cdot 3$ & $\leq 0.02$ & 1,2915 \\
\hline Incomplete primary & 730 & $58 \cdot 0$ & $54 \cdot 0,61 \cdot 8$ & 1458 & $50 \cdot 9$ & $47 \cdot 3,54 \cdot 5$ & 1140 & $49 \cdot 0$ & $45 \cdot 7,52 \cdot 3$ & 814 & $44 \cdot 3$ & $39 \cdot 7,49 \cdot 1$ & 662 & $37 \cdot 1$ & $32 \cdot 9,41 \cdot 5$ & $44 \cdot 9$ & $\leq 0 \cdot 00$ & 1,296 \\
\hline No education & 356 & $70 \cdot 8$ & $65 \cdot 6,75 \cdot 5$ & 699 & $60 \cdot 4$ & $55 \cdot 9,64 \cdot 8$ & 399 & $59 \cdot 5$ & $54 \cdot 0,64 \cdot 7$ & 160 & $60 \cdot 8$ & $51 \cdot 9,69 \cdot 1$ & 136 & $56 \cdot 6$ & $47 \cdot 2,65 \cdot 6$ & $6 \cdot 9$ & $\leq 0.01$ & 1,1271 \\
\hline \multicolumn{19}{|l|}{ Wealth quintile } \\
\hline Richest & 343 & $7 \cdot 2$ & $4 \cdot 7,10 \cdot 8$ & 789 & $7 \cdot 5$ & $5 \cdot 2,10 \cdot 7$ & 567 & $5 \cdot 3$ & $3 \cdot 2,8 \cdot 6$ & 528 & $7 \cdot 3$ & $4 \cdot 7,11 \cdot 1$ & 310 & $1 \cdot 4$ & $0.5,3 \cdot 9$ & $7 \cdot 0$ & $\leq 0 \cdot 01$ & 1,1734 \\
\hline Richer & 520 & $15 \cdot 7$ & $12 \cdot 6,19 \cdot 4$ & 1179 & $11 \cdot 5$ & $9 \cdot 2,14 \cdot 3$ & 873 & $10 \cdot 3$ & $7 \cdot 9,13 \cdot 4$ & 674 & $12 \cdot 2$ & $8 \cdot 9,16 \cdot 5$ & 582 & $5 \cdot 5$ & $3 \cdot 5,8 \cdot 5$ & $13 \cdot 6$ & $\leq 0.00$ & 1,2414 \\
\hline Middle & 657 & $28 \cdot 9$ & $25 \cdot 0,33 \cdot 2$ & 1413 & $24 \cdot 5$ & $21 \cdot 8,27 \cdot 5$ & 1103 & $22 \cdot 1$ & $19 \cdot 3,25 \cdot 2$ & 1098 & $23 \cdot 2$ & $19 \cdot 8,27 \cdot 0$ & 893 & $9 \cdot 0$ & $6 \cdot 8,11 \cdot 6$ & $54 \cdot 7$ & $\leq 0.00$ & 1,2952 \\
\hline Poorer & 868 & $44 \cdot 1$ & $40 \cdot 6,47 \cdot 7$ & 1534 & $39 \cdot 0$ & $35 \cdot 9,42 \cdot 2$ & 1181 & $37 \cdot 6$ & $34 \cdot 4,41 \cdot 0$ & 1159 & $42 \cdot 4$ & $38 \cdot 3,46 \cdot 3$ & 1134 & $22 \cdot 0$ & $19 \cdot 3,25 \cdot 0$ & $49 \cdot 6$ & $\leq 0.00$ & 1,3020 \\
\hline Poorest & 1053 & $61 \cdot 1$ & $57 \cdot 7,64 \cdot 3$ & 1807 & $54 \cdot 8$ & $51 \cdot 2,58 \cdot 3$ & 1445 & $55 \cdot 4$ & $52 \cdot 7,58 \cdot 1$ & 559 & $51 \cdot 7$ & $46 \cdot 1,57 \cdot 3$ & 1393 & $42 \cdot 9$ & $39 \cdot 3,46 \cdot 6$ & $43 \cdot 1$ & $\leq 0.00$ & 1,201 \\
\hline
\end{tabular}

*Using the 2006 WHO Child Growth Standards ${ }^{(24)}$

tStatistical significance of trends across years within each subgroup $(P \leq 0.05)$ 
Table 3 Prevalence estimates for overweight (weight-for-height $>+2$ sD above the median of the reference population*) in males (aged 0-59 months) and test for trend over time by sociodemographic factors. Peru Demographic and Health Surveys, 1991-2011

\begin{tabular}{|c|c|c|c|c|c|c|c|c|c|c|c|c|c|c|c|c|c|c|}
\hline \multirow[b]{2}{*}{ Variable } & \multicolumn{3}{|c|}{$1991-92$} & \multicolumn{3}{|c|}{1996} & \multicolumn{3}{|c|}{2000} & \multicolumn{3}{|c|}{ 2007-08 } & \multicolumn{3}{|c|}{2011} & \multirow[b]{2}{*}{ Wald $(F)$} & \multirow[b]{2}{*}{$P \dagger$} & \multirow[b]{2}{*}{ df } \\
\hline & $n$ & $\%$ & $95 \% \mathrm{Cl}$ & $n$ & $\%$ & $95 \% \mathrm{Cl}$ & $n$ & $\%$ & $95 \% \mathrm{Cl}$ & $n$ & $\%$ & $95 \% \mathrm{Cl}$ & $n$ & $\%$ & $95 \% \mathrm{Cl}$ & & & \\
\hline Total both sexes & 7098 & $9 \cdot 3$ & $8 \cdot 5,10 \cdot 2$ & 13516 & $10 \cdot 0$ & $9 \cdot 2,10 \cdot 8$ & 10515 & $11 \cdot 9$ & $11 \cdot 0,12 \cdot 7$ & 7374 & $10 \cdot 0$ & $9 \cdot 0,11 \cdot 2$ & 8063 & $8 \cdot 8$ & $7 \cdot 8,10 \cdot 0$ & 0.4 & $\leq 0.54$ & 1,5147 \\
\hline $\begin{array}{l}\text { Total males } \\
\text { Age (imonths) }\end{array}$ & \multicolumn{7}{|c|}{ Age (imonths) } & & $11 \cdot 4,13 \cdot 8$ & 3723 & $10 \cdot 2$ & $8 \cdot 7,11 \cdot 9$ & 4113 & $10 \cdot 4$ & $8 \cdot 9,12 \cdot 2$ & $0 \cdot 0$ & $\leq 0.92$ & 1,5147 \\
\hline $0-5$ & 504 & $12 \cdot 5$ & $9 \cdot 7,15 \cdot 9$ & 884 & $17 \cdot 2$ & $14 \cdot 2,20 \cdot 8$ & 586 & $17 \cdot 4$ & $14 \cdot 1,21 \cdot 4$ & 440 & $22 \cdot 5$ & $17 \cdot 6,28 \cdot 3$ & 453 & $14 \cdot 4$ & $10 \cdot 6,19 \cdot 3$ & $1 \cdot 8$ & $\leq 0 \cdot 18$ & 1,2854 \\
\hline $6-11$ & 483 & $8 \cdot 7$ & $6 \cdot 2,12 \cdot 2$ & 921 & $11 \cdot 6$ & $9 \cdot 3,14 \cdot 5$ & 611 & $12 \cdot 5$ & $9 \cdot 5,16 \cdot 2$ & 433 & $11 \cdot 3$ & $7 \cdot 0,17 \cdot 7$ & 456 & $10 \cdot 8$ & $7 \cdot 5,15 \cdot 3$ & $0 \cdot 3$ & $\leq 0.57$ & 1,2925 \\
\hline $12-23$ & 837 & $9 \cdot 5$ & $7 \cdot 5,12 \cdot 0$ & 1665 & $9 \cdot 6$ & $8 \cdot 0,11 \cdot 5$ & 1333 & $11 \cdot 3$ & $9 \cdot 2,13 \cdot 8$ & 803 & $7 \cdot 8$ & $5 \cdot 6,10 \cdot 7$ & 922 & $10 \cdot 9$ & $8 \cdot 0,14 \cdot 6$ & $0 \cdot 1$ & $\leq 0.77$ & 1,4029 \\
\hline $24-35$ & 748 & $8 \cdot 5$ & $6 \cdot 6,10 \cdot 9$ & 1338 & $10 \cdot 7$ & $8 \cdot 7,13 \cdot 0$ & 1036 & $10 \cdot 5$ & $8 \cdot 3,13 \cdot 2$ & 764 & $8 \cdot 2$ & $5 \cdot 4,12 \cdot 4$ & 947 & $7 \cdot 5$ & $5 \cdot 4,10 \cdot 4$ & $1 \cdot 8$ & $\leq 0 \cdot 18$ & 1,4073 \\
\hline $36-47$ & 612 & $11 \cdot 5$ & $9 \cdot 1,14 \cdot 4$ & 1104 & $10 \cdot 4$ & $8 \cdot 3,13 \cdot 0$ & 960 & $13 \cdot 9$ & $11 \cdot 2,17 \cdot 2$ & 707 & $7 \cdot 2$ & $4 \cdot 9,10 \cdot 7$ & 724 & 8.9 & $6 \cdot 3,12 \cdot 5$ & $2 \cdot 9$ & $\leq 0.09$ & 1,4137 \\
\hline $48-59$ & 496 & $8 \cdot 4$ & $6 \cdot 1,11 \cdot 5$ & 934 & $8 \cdot 3$ & $6 \cdot 3,10 \cdot 8$ & 844 & $12 \cdot 0$ & $9 \cdot 5,15 \cdot 2$ & 577 & $9 \cdot 5$ & $6 \cdot 1,14 \cdot 6$ & 611 & $12 \cdot 9$ & $8 \cdot 8,18 \cdot 6$ & $3 \cdot 3$ & $\leq 0.07$ & 1,3993 \\
\hline \multicolumn{19}{|l|}{ Residence } \\
\hline Urban & 2255 & $10 \cdot 3$ & $8 \cdot 9,11 \cdot 9$ & 4123 & $13 \cdot 2$ & $11 \cdot 8,14 \cdot 8$ & 2910 & $14 \cdot 6$ & $12 \cdot 7,16 \cdot 7$ & 2228 & $11 \cdot 6$ & $9 \cdot 4,14 \cdot 2$ & 2728 & $13 \cdot 4$ & $11 \cdot 3,15 \cdot 8$ & $1 \cdot 4$ & $\leq 0.23$ & 1,3333 \\
\hline Rural & 1425 & 8.9 & $7 \cdot 3,10 \cdot 8$ & 2728 & $7 \cdot 7$ & $6 \cdot 6,9 \cdot 0$ & 2460 & $10 \cdot 1$ & $8 \cdot 9,11 \cdot 5$ & 1496 & $8 \cdot 0$ & $6 \cdot 6,9 \cdot 8$ & 1385 & $4 \cdot 7$ & $3 \cdot 7,6 \cdot 1$ & $8 \cdot 7$ & $\leq 0.00$ & 1,1943 \\
\hline \multicolumn{19}{|l|}{ Geographic region } \\
\hline Coast & 1754 & $11 \cdot 2$ & $9 \cdot 6,13 \cdot 1$ & 3110 & $15 \cdot 6$ & $13 \cdot 8,17 \cdot 5$ & 2326 & $17 \cdot 0$ & $14 \cdot 7,19 \cdot 6$ & 1617 & $14 \cdot 2$ & $11 \cdot 4,17 \cdot 5$ & 1639 & $16 \cdot 5$ & $13 \cdot 8,19 \cdot 7$ & $4 \cdot 8$ & $\leq 0.03$ & 1,2255 \\
\hline Andes & 500 & $7 \cdot 2$ & $5 \cdot 3,9 \cdot 7$ & 2694 & $8 \cdot 3$ & $7 \cdot 2,9 \cdot 6$ & 2563 & $9 \cdot 9$ & $8 \cdot 8,11 \cdot 2$ & 1615 & $7 \cdot 9$ & $6 \cdot 5,9 \cdot 6$ & 1166 & $4 \cdot 6$ & $3 \cdot 5,6 \cdot 0$ & $8 \cdot 4$ & $\leq 0 \cdot 00$ & 1,1890 \\
\hline Amazon basin & 1425 & $8 \cdot 9$ & $7 \cdot 3,10 \cdot 8$ & 1047 & $4 \cdot 4$ & $3 \cdot 3,5 \cdot 9$ & 480 & $4 \cdot 8$ & $3 \cdot 5,6 \cdot 4$ & 491 & $4 \cdot 4$ & $2 \cdot 8,6 \cdot 7$ & 502 & $3 \cdot 8$ & $2 \cdot 6,5 \cdot 5$ & $21 \cdot 5$ & $\leq 0.00$ & 1,1096 \\
\hline \multicolumn{19}{|l|}{ Maternal education } \\
\hline Complete secondary/higher & 1151 & $11 \cdot 0$ & $9 \cdot 1,13 \cdot 2$ & 2181 & $14 \cdot 0$ & $12 \cdot 2,16 \cdot 1$ & 1945 & $14 \cdot 2$ & $12 \cdot 6,16 \cdot 6$ & 1651 & $12 \cdot 6$ & $10 \cdot 2,15 \cdot 4$ & 2041 & $15 \cdot 3$ & $13 \cdot 0,18 \cdot 0$ & $2 \cdot 3$ & $\leq 0 \cdot 13$ & 1,4119 \\
\hline Incomplete secondary & 663 & $10 \cdot 1$ & $8 \cdot 0,12 \cdot 8$ & 1251 & $11 \cdot 0$ & $8 \cdot 9,13 \cdot 6$ & 845 & $13 \cdot 2$ & $10 \cdot 6,16 \cdot 3$ & 628 & $10 \cdot 4$ & $7 \cdot 5,14 \cdot 2$ & 688 & $6 \cdot 8$ & $4 \cdot 7,9 \cdot 7$ & $5 \cdot 5$ & $\leq 0.02$ & 1,3289 \\
\hline Complete primary & 738 & $8 \cdot 0$ & $6 \cdot 2,10 \cdot 2$ & 1215 & $9 \cdot 0$ & $7 \cdot 2,11 \cdot 0$ & 899 & $11 \cdot \overline{7}$ & $9 \cdot 4,14 \cdot 5$ & 537 & $8 \cdot 1$ & $5 \cdot 7,11 \cdot 4$ & 544 & $5 \cdot 0$ & $3 \cdot 2,7 \cdot 7$ & $1 \cdot 9$ & $\leq 0.17$ & 1,2915 \\
\hline Incomplete primary & 764 & $9 \cdot 3$ & $7 \cdot 4,11 \cdot 6$ & 1562 & $9 \cdot 4$ & $7 \cdot 8,11 \cdot 3$ & 1265 & $11 \cdot 1$ & $9 \cdot 3,13 \cdot 2$ & 744 & $6 \cdot 5$ & $4 \cdot 7,8 \cdot 7$ & 708 & $5 \cdot 4$ & $3 \cdot 6,8 \cdot 1$ & $1 \cdot 3$ & $\leq 0.01$ & 1, 2959 \\
\hline No education & 364 & $9 \cdot 9$ & $7 \cdot 2,13 \cdot 4$ & 643 & $8 \cdot 7$ & $6 \cdot 4,11 \cdot 7$ & 416 & $9 \cdot 5$ & $6 \cdot 9,13 \cdot 0$ & 164 & $8 \cdot 9$ & $5 \cdot 0,15 \cdot 2$ & 132 & $3 \cdot 3$ & $1 \cdot 2,8 \cdot 7$ & $2 \cdot 6$ & $\leq 0 \cdot 10$ & 1,1267 \\
\hline \multicolumn{19}{|l|}{ Wealth quintile } \\
\hline Richest & 341 & $12 \cdot 3$ & $8.9,16.9$ & 726 & $14 \cdot 5$ & $11 \cdot 2,18 \cdot 5$ & 645 & $14 \cdot 5$ & $10 \cdot 9,19 \cdot 0$ & 711 & $17 \cdot 6$ & $13 \cdot 1,23 \cdot 3$ & 532 & $20 \cdot 1$ & $15 \cdot 1,26 \cdot 4$ & $5 \cdot 5$ & $\leq 0.02$ & 1,1730 \\
\hline Richer & 577 & $9 \cdot 9$ & $7 \cdot 4,13 \cdot 0$ & 1147 & $15 \cdot 0$ & $12 \cdot 4,17 \cdot 9$ & 857 & $15 \cdot 3$ & $12 \cdot 2,19 \cdot 0$ & 646 & $11 \cdot 3$ & $8 \cdot 0,15 \cdot 7$ & 723 & $14 \cdot 9$ & $11 \cdot 2,19 \cdot 7$ & $1 \cdot 1$ & $\leq 0.29$ & 1,2408 \\
\hline Middle & 730 & $10 \cdot 0$ & $7 \cdot 9,12 \cdot 6$ & 1444 & $12 \cdot 2$ & $10 \cdot 2,14 \cdot 6$ & 1063 & $15 \cdot 8$ & $13 \cdot 0,19 \cdot 0$ & 903 & $8 \cdot 0$ & $6 \cdot 2,10 \cdot 4$ & 953 & $11 \cdot 1$ & $8 \cdot 4,14 \cdot 6$ & 0.3 & $\leq 0.57$ & 1,2949 \\
\hline Poorer & 958 & $9 \cdot 9$ & $8 \cdot 1,12 \cdot 0$ & 1683 & $10 \cdot 7$ & $8 \cdot 9,12 \cdot 7$ & 1314 & $10 \cdot 2$ & $8 \cdot 5,12 \cdot 3$ & 1014 & $6 \cdot 8$ & $5 \cdot 3,8 \cdot 7$ & 934 & $6 \cdot 6$ & $4 \cdot 7,9 \cdot 2$ & $9 \cdot 4$ & $\leq 0.00$ & 1,3015 \\
\hline Poorest & 1073 & $8 \cdot 7$ & $7 \cdot 2,10 \cdot 5$ & 1851 & $6 \cdot 6$ & $5 \cdot 6,7 \cdot 9$ & 1492 & $9 \cdot 8$ & $8 \cdot 4,11 \cdot 5$ & 451 & $8 \cdot 7$ & $6 \cdot 3,11 \cdot 9$ & 971 & $4 \cdot 8$ & $3 \cdot 7,6 \cdot 3$ & $3 \cdot 3$ & $\leq 0.07$ & 1,2010 \\
\hline
\end{tabular}

*Using the 2006 WHO Child Growth Standards(24).

tStatistical significance of trends across years within each subgroup $(P \leq 0.05)$. 
Table 4 Prevalence estimates for overweight (weight-for-height $>+2$ sD above the median of the reference population*) in females (aged 0-59 months) and test for trend over time by sociodemographic factors. Peru Demographic and Health Surveys, 1991-2011

\begin{tabular}{|c|c|c|c|c|c|c|c|c|c|c|c|c|c|c|c|c|c|c|}
\hline \multirow[b]{2}{*}{ Variable } & \multicolumn{3}{|c|}{ 1991-92 } & \multicolumn{3}{|c|}{1996} & \multicolumn{3}{|c|}{2000} & \multicolumn{3}{|c|}{$2007-08$} & \multicolumn{3}{|c|}{2011} & \multirow[b]{2}{*}{ Wald $(F)$} & \multirow[b]{2}{*}{$P+$} & \multirow[b]{2}{*}{ df } \\
\hline & $n$ & $\%$ & $95 \% \mathrm{Cl}$ & $n$ & $\%$ & $95 \% \mathrm{Cl}$ & $n$ & $\%$ & $95 \% \mathrm{Cl}$ & $n$ & $\%$ & $95 \% \mathrm{Cl}$ & $n$ & $\%$ & $95 \% \mathrm{Cl}$ & & & \\
\hline Total both sexes & 7098 & $9 \cdot 3$ & $8 \cdot 5,10 \cdot 2$ & 13516 & $10 \cdot 0$ & $9 \cdot 2,10 \cdot 8$ & 10515 & $11 \cdot 9$ & $11 \cdot 0,12 \cdot 7$ & 7374 & $10 \cdot 0$ & $9 \cdot 0,11 \cdot 2$ & 8063 & $8 \cdot 8$ & $7 \cdot 8,10 \cdot 0$ & 0.4 & $\leq 0.54$ & 1,5147 \\
\hline Total females & 3419 & $8 \cdot 8$ & $7 \cdot 8,9 \cdot 9$ & 6665 & 8.9 & $8 \cdot 0,10 \cdot 0$ & 5145 & $11 \cdot 2$ & $10 \cdot 1,12 \cdot 3$ & 3650 & $9 \cdot 9$ & $8 \cdot 5,11 \cdot 5$ & 3950 & $7 \cdot 2$ & $6 \cdot 1,8 \cdot 4$ & $1 \cdot 3$ & $\leq 0.26$ & 1,5147 \\
\hline \multicolumn{19}{|l|}{ Age (months) } \\
\hline $0-5$ & 265 & $12 \cdot 9$ & $9 \cdot 2,17 \cdot 7$ & 452 & $14 \cdot 0$ & $10 \cdot 3,18 \cdot 7$ & 364 & $18 \cdot 9$ & $14 \cdot 3,24 \cdot 5$ & 245 & $24 \cdot 8$ & $18 \cdot 3,32 \cdot 7$ & 310 & $14 \cdot 5$ & $10 \cdot 0,20 \cdot 7$ & $2 \cdot 7$ & $\leq 0 \cdot 10$ & 1,2854 \\
\hline $6-11$ & 264 & $10 \cdot 9$ & $7 \cdot 4,15 \cdot 7$ & 498 & $10 \cdot 1$ & $7 \cdot 2,14 \cdot 1$ & 391 & $12 \cdot 6$ & $9 \cdot 1,17 \cdot 2$ & 293 & $16 \cdot 1$ & $10 \cdot 9,23 \cdot 1$ & 361 & $8 \cdot 0$ & $4 \cdot 7,13 \cdot 4$ & 0.0 & $\leq 0.94$ & 1,2925 \\
\hline $12-23$ & 509 & $8 \cdot 1$ & $5 \cdot 8,11 \cdot 2$ & 1075 & $10 \cdot 3$ & $7 \cdot 9,13 \cdot 3$ & 833 & $13 \cdot 4$ & $10 \cdot 6,16 \cdot 8$ & 661 & $10 \cdot 7$ & $7 \cdot 3,15 \cdot 5$ & 671 & $6 \cdot 3$ & $4 \cdot 7,13 \cdot 4$ & $0 \cdot 8$ & $\leq 0.36$ & 1,4029 \\
\hline $24-35$ & 630 & $10 \cdot 1$ & $7 \cdot 9,12 \cdot 8$ & 1312 & $8 \cdot 4$ & $6 \cdot 7,10 \cdot 5$ & 1039 & $9 \cdot 1$ & $7 \cdot 1,11 \cdot 5$ & 711 & $6 \cdot 7$ & $4 \cdot 4,10 \cdot 1$ & 821 & $6 \cdot 3$ & $4 \cdot 2,9 \cdot 2$ & $4 \cdot 8$ & $\leq 0.03$ & 1,4073 \\
\hline $36-47$ & 891 & $9 \cdot 1$ & $7 \cdot 3,11 \cdot 3$ & 1637 & $8 \cdot 0$ & $6 \cdot 5,9 \cdot 9$ & 1181 & $8 \cdot 8$ & $7 \cdot 0,11 \cdot 0$ & 845 & $8 \cdot 6$ & $6 \cdot 1,12 \cdot 0$ & 843 & $6 \cdot 9$ & $4 \cdot 9,9 \cdot 6$ & 0.8 & $\leq 0 \cdot 36$ & 1,4137 \\
\hline $48-59$ & 861 & $6 \cdot 0$ & $4 \cdot 5,7 \cdot 9$ & 1692 & $7 \cdot 6$ & $6 \cdot 2,9 \cdot 3$ & 1338 & $10 \cdot 9$ & $8 \cdot 9,13 \cdot 3$ & 895 & $6 \cdot 9$ & $4 \cdot 8,9 \cdot 8$ & 944 & $6 \cdot 1$ & $4 \cdot 3,8 \cdot 7$ & $0 \cdot 0$ & $\leq 0.89$ & 1,3993 \\
\hline \multicolumn{19}{|l|}{ Residence } \\
\hline Urban & 2103 & $9 \cdot 5$ & $8 \cdot 3,10 \cdot 9$ & 4039 & $10 \cdot 1$ & $8 \cdot 8,11 \cdot 4$ & 2862 & $12 \cdot 6$ & $11 \cdot 0,14 \cdot 4$ & 2166 & $11 \cdot 4$ & $9 \cdot 3,13 \cdot 9$ & 2563 & 8.9 & $7 \cdot 4,10 \cdot 7$ & $0 \cdot 0$ & $\leq 0.96$ & 1,3333 \\
\hline Rural & 1316 & $7 \cdot 6$ & $6 \cdot 1,9 \cdot 5$ & 2626 & $7 \cdot 1$ & $5 \cdot 7,8 \cdot 9$ & 2283 & $9 \cdot 3$ & $8 \cdot 0,10 \cdot 9$ & 1484 & $7 \cdot 7$ & $6 \cdot 2,9 \cdot 6$ & 1387 & $4 \cdot 0$ & $2 \cdot 9,5 \cdot 4$ & $6 \cdot 0$ & $\leq 0.02$ & 1,1943 \\
\hline \multicolumn{19}{|l|}{ Geographic region } \\
\hline Coast & 1652 & $10 \cdot 2$ & $8 \cdot 7,11 \cdot 8$ & 3139 & $11 \cdot 5$ & $10 \cdot 0,13 \cdot 2$ & 2362 & $14 \cdot 0$ & $12 \cdot 1,16 \cdot 1$ & 1577 & $13 \cdot 6$ & $10 \cdot 8,16 \cdot 9$ & 1574 & $12 \cdot 3$ & $10 \cdot 0,15 \cdot 1$ & $3 \cdot 7$ & $\leq 0.06$ & 1,2255 \\
\hline Andes & 451 & $7 \cdot 2$ & $5 \cdot 2,9 \cdot 9$ & 2584 & $7 \cdot 7$ & $6 \cdot 3,9 \cdot 5$ & 2351 & $9 \cdot 6$ & $8 \cdot 3,11 \cdot 1$ & 1608 & $8 \cdot 2$ & $6 \cdot 7,10 \cdot 0$ & 1156 & 3.9 & $2 \cdot 8,5 \cdot 2$ & $6 \cdot 4$ & $\leq 0.01$ & 1,1890 \\
\hline Amazon basin & 1316 & $7 \cdot 6$ & $6 \cdot 1,9 \cdot 5$ & 942 & $3 \cdot 6$ & $2 \cdot 5,5 \cdot 2$ & 432 & $4 \cdot 0$ & $2 \cdot 8,5 \cdot 7$ & 465 & $3 \cdot 4$ & $2 \cdot 2,5 \cdot 3$ & 466 & $2 \cdot 9$ & $1 \cdot 9,4 \cdot 6$ & $18 \cdot 1$ & $\leq 0.00$ & 1,1096 \\
\hline \multicolumn{19}{|l|}{ Maternal education } \\
\hline Complete secondary/higher & 1115 & $9 \cdot 7$ & $7 \cdot 9,11 \cdot 7$ & 2205 & $11 \cdot 0$ & $9 \cdot 4,13 \cdot 0$ & 1876 & $12 \cdot 1$ & $10 \cdot 2,14 \cdot 3$ & 1596 & $12 \cdot 3$ & $9 \cdot 9,15 \cdot 3$ & 1953 & $9 \cdot 6$ & $7 \cdot 9,11 \cdot 7$ & $0 \cdot 0$ & $\leq 0.95$ & 1,4119 \\
\hline Incomplete secondary & 578 & $6 \cdot 7$ & $4 \cdot 8,9 \cdot 1$ & 1185 & $7 \cdot 6$ & $5 \cdot 8,9 \cdot 8$ & 859 & $13 \cdot 1$ & $10 \cdot 3,16 \cdot 4$ & 593 & $7 \cdot 4$ & $4 \cdot 9,10 \cdot 9$ & 701 & $5 \cdot 5$ & $3 \cdot 3,8 \cdot 9$ & $28 \cdot 1$ & $\leq 0.00$ & 1,3289 \\
\hline Complete primary & 652 & $8 \cdot 8$ & $6 \cdot 8,11 \cdot 2$ & 1145 & $7 \cdot 1$ & $5 \cdot 6,8 \cdot 9$ & 878 & $10 \cdot 1$ & $7 \cdot 9,12 \cdot 8$ & 490 & $10 \cdot 2$ & $7 \cdot 1,14 \cdot 3$ & 500 & $4 \cdot 2$ & $2 \cdot 5,6 \cdot 9$ & $1 \cdot 3$ & $\leq 0.26$ & 1,2915 \\
\hline Incomplete primary & 725 & $9 \cdot 1$ & $7 \cdot 2,11 \cdot 5$ & 1449 & $8 \cdot 6$ & $7 \cdot 0,10 \cdot 5$ & 1132 & $9 \cdot 5$ & $7 \cdot 8,11 \cdot 5$ & 814 & $7 \cdot 7$ & $5 \cdot 7,10 \cdot 3$ & 662 & $4 \cdot 6$ & $3 \cdot 2,6 \cdot 7$ & $6 \cdot 0$ & $\leq 0.01$ & 1,2959 \\
\hline No education & 350 & $8 \cdot 9$ & $6 \cdot 3,12 \cdot 3$ & 681 & $8 \cdot 2$ & $6 \cdot 1,10 \cdot 8$ & 400 & $9 \cdot 7$ & $6 \cdot 9,13 \cdot 4$ & 157 & $5 \cdot 3$ & $2 \cdot 6,10 \cdot 6$ & 135 & $4 \cdot 3$ & $1 \cdot 9,9 \cdot 5$ & $2 \cdot 4$ & $\leq 0 \cdot 12$ & 1,1267 \\
\hline \multicolumn{19}{|l|}{ Wealth quintile } \\
\hline Richest & 342 & $9 \cdot 3$ & $6 \cdot 5,13 \cdot 2$ & 783 & $13 \cdot 0$ & $10 \cdot 0,16 \cdot 7$ & 562 & $16 \cdot 5$ & $12 \cdot 4,21 \cdot 7$ & 707 & $15 \cdot 4$ & $11 \cdot 4,20 \cdot 5$ & 510 & $15 \cdot 2$ & $10 \cdot 7,21 \cdot 1$ & 2.9 & $\leq 0.09$ & 1,1730 \\
\hline Richer & 516 & $10 \cdot 3$ & $7 \cdot 7,13 \cdot 5$ & 1177 & $10 \cdot 1$ & $8 \cdot 0,12 \cdot 7$ & 869 & $14 \cdot 0$ & $11 \cdot 0,17 \cdot 6$ & 652 & $12 \cdot 9$ & $9 \cdot 1,18 \cdot 1$ & 701 & 11.5 & $8 \cdot 3,15 \cdot 7$ & $1 \cdot 1$ & $\leq 0.29$ & 1,2408 \\
\hline Middle & 657 & $10 \cdot 4$ & $8 \cdot 2,12 \cdot 3$ & 1402 & $8 \cdot 4$ & $6 \cdot 7,10 \cdot 5$ & 1099 & $10 \cdot 7$ & $8 \cdot 5,13 \cdot 4$ & 926 & $7 \cdot 5$ & $5 \cdot 5,10 \cdot 2$ & 863 & $5 \cdot 8$ & $4 \cdot 1,8 \cdot 0$ & $7 \cdot 4$ & $\leq 0.01$ & 1,2949 \\
\hline Poorer & 862 & $7 \cdot 4$ & $5 \cdot 8,9 \cdot 4$ & 1525 & $7 \cdot 8$ & $6 \cdot 4,9 \cdot 6$ & 1178 & $9 \cdot 5$ & $7 \cdot 7,11 \cdot 6$ & 919 & $7 \cdot 0$ & $5 \cdot 3,9 \cdot 0$ & 886 & $4 \cdot 4$ & $3 \cdot 0,6 \cdot 5$ & $5 \cdot 2$ & $\leq 0.02$ & 1,3015 \\
\hline Poores & 1041 & $8 \cdot 0$ & $6 \cdot 5,9 \cdot 8$ & 1778 & $7 \cdot 7$ & $6 \cdot 4,9 \cdot 3$ & 1437 & $9 \cdot 1$ & $7 \cdot 6,10 \cdot 7$ & 447 & $7 \cdot 8$ & $5 \cdot 5,10 \cdot 9$ & 990 & $3 \cdot 7$ & $2 \cdot 7,5 \cdot 0$ & $9 \cdot 8$ & $\leq 0.00$ & 1,2010 \\
\hline
\end{tabular}

*Using the 2006 WHO Child Growth Standards ${ }^{(24)}$

tStatistical significance of trends across years within each subgroup $(P \leq 0.05)$. 
Table 5 Prevalence estimates for stunting (height-for-age $<-2$ SD below the median of the reference population*) and overweight (weight-for-height $>+2$ sD above the median of the reference population*) in children (aged 0-59 months) for selectedt sociodemographic factors. Peru Demographic and Health Surveys, 1991-2011

\begin{tabular}{|c|c|c|c|c|c|c|c|c|c|c|c|c|c|c|c|}
\hline & \multicolumn{3}{|c|}{$1991-92$} & \multicolumn{3}{|c|}{1996} & \multicolumn{3}{|c|}{2000} & \multicolumn{3}{|c|}{ 2007-08 } & \multicolumn{3}{|c|}{2011} \\
\hline & $n$ & $\%$ & $95 \% \mathrm{Cl} \%$ & $n$ & $\%$ & $95 \% \mathrm{Cl} \%$ & $n$ & $\%$ & $95 \% \mathrm{Cl}$ & $n$ & $\%$ & $95 \% \mathrm{Cl}$ & $n$ & $\%$ & $95 \% \mathrm{Cl}$ \\
\hline \multicolumn{16}{|l|}{ Stunting } \\
\hline \multicolumn{16}{|l|}{ Residence } \\
\hline Urban & 4369 & $26 \cdot 4$ & $24 \cdot 5,28 \cdot 5$ & 8200 & $21 \cdot 1$ & $19 \cdot 5,22 \cdot 8$ & 5877 & $18 \cdot 2$ & $16 \cdot 6,20 \cdot 0$ & 4414 & $16 \cdot 2$ & $14 \cdot 4,18 \cdot 1$ & 5300 & $10 \cdot 1$ & $8 \cdot 8,11 \cdot 5$ \\
\hline Rural & 2769 & $51 \cdot 9$ & $51 \cdot 9,57 \cdot 4$ & 5411 & $48 \cdot 0$ & $45 \cdot 6,50 \cdot 4$ & 4774 & $47 \cdot 4$ & $45 \cdot 3,49 \cdot 4$ & 2989 & $45 \cdot 1$ & $42 \cdot 2,48 \cdot 1$ & 2773 & $36 \cdot 9$ & $34 \cdot 1,39 \cdot 8$ \\
\hline \multicolumn{16}{|l|}{ Geographic region } \\
\hline Coast & 3414 & $23 \cdot 8$ & $21 \cdot 7,26 \cdot 1$ & 6272 & $18 \cdot 0$ & $16 \cdot 2,19 \cdot 9$ & 4716 & $15 \cdot 9$ & $13 \cdot 9,18 \cdot 1$ & 3209 & $15 \cdot 2$ & $13 \cdot 1,17 \cdot 6$ & 3223 & $7 \cdot 6$ & $6 \cdot 1,9 \cdot 3$ \\
\hline Andes & 955 & $35 \cdot 7$ & $31 \cdot 4,40 \cdot 3$ & 5338 & $44 \cdot 9$ & $42 \cdot 6,47 \cdot 2$ & 4950 & $44 \cdot 9$ & $42 \cdot 9,46 \cdot 9$ & 3236 & $40 \cdot 0$ & $36 \cdot 7,43 \cdot 5$ & 2324 & $29 \cdot 7$ & $27 \cdot 0,32 \cdot 5$ \\
\hline Amazon & 2769 & $54 \cdot 7$ & $51 \cdot 9,57 \cdot 4$ & 2001 & $40 \cdot 0$ & $26 \cdot 9,43 \cdot 3$ & 919 & $37 \cdot 8$ & $34 \cdot 2,41 \cdot 6$ & 959 & $29 \cdot 1$ & $25 \cdot 5,32 \cdot 9$ & 967 & $25 \cdot 1$ & $22 \cdot 1,28 \cdot 2$ \\
\hline \multicolumn{16}{|l|}{ Maternal education } \\
\hline Complete secondary/higher & 2277 & $16 \cdot 6$ & $14 \cdot 8,18 \cdot 6$ & 4402 & $12 \cdot 7$ & $11 \cdot 3,14 \cdot 3$ & 3858 & $13 \cdot 0$ & $11 \cdot 6,14 \cdot 6$ & 3267 & $12 \cdot 8$ & $11 \cdot 1,14 \cdot 6$ & 4005 & $7 \cdot 7$ & $6 \cdot 6,8 \cdot 9$ \\
\hline Incomplete secondary & 1244 & $30 \cdot 4$ & $27 \cdot 4,33 \cdot 5$ & 2446 & $26 \cdot 4$ & $24 \cdot 2,28 \cdot 7$ & 1708 & $26 \cdot 5$ & $24 \cdot 0,29 \cdot 2$ & 1223 & $26 \cdot 8$ & $23 \cdot 5,30 \cdot 4$ & 1389 & $18 \cdot 1$ & $15 \cdot 5,21 \cdot 0$ \\
\hline Complete primary & 1397 & $43 \cdot 1$ & $40 \cdot 3,46 \cdot 0$ & 2381 & $36 \cdot 5$ & $33 \cdot 6,39 \cdot 4$ & 1787 & $39 \cdot 5$ & $36 \cdot 6,42 \cdot 3$ & 1029 & $37 \cdot 3$ & $33 \cdot 6,41 \cdot 1$ & 1043 & $32 \cdot 8$ & $29 \cdot 4,36 \cdot 3$ \\
\hline Incomplete primary & 1467 & $55 \cdot 1$ & $52 \cdot 1,58 \cdot 1$ & 3031 & $49 \cdot 3$ & $46 \cdot 6,52 \cdot 0$ & 2413 & $49 \cdot 1$ & $46 \cdot 6,51 \cdot 1$ & 1560 & $46 \cdot 8$ & $43 \cdot 4,50 \cdot 3$ & 1370 & $37 \cdot 4$ & $34 \cdot 1,40 \cdot 9$ \\
\hline No education & 724 & $67 \cdot 1$ & $63 \cdot 2,70 \cdot 8$ & 1349 & $56 \cdot 3$ & $52 \cdot 7,59 \cdot 8$ & 820 & 58.0 & $54 \cdot 0,61 \cdot 9$ & 324 & $62 \cdot 5$ & $56 \cdot 2,68 \cdot 4$ & 267 & $53 \cdot 8$ & $46 \cdot 6,60 \cdot 8$ \\
\hline \multicolumn{16}{|l|}{ Overweight } \\
\hline \multicolumn{16}{|l|}{ Geographic region } \\
\hline Coast & 3406 & $10 \cdot 7$ & $9 \cdot 5,12 \cdot 0$ & 6249 & $13 \cdot 5$ & $12 \cdot 3,14 \cdot 8$ & 4688 & $15 \cdot 5$ & $14 \cdot 0,17 \cdot 1$ & 3194 & $13 \cdot 9$ & $11 \cdot 8,16 \cdot 3$ & 3213 & $14 \cdot 4$ & $12 \cdot 6,16 \cdot 5$ \\
\hline Andes & 951 & $7 \cdot 2$ & $5 \cdot 7,9 \cdot 1$ & 5278 & $8 \cdot 0$ & $7 \cdot 0,9 \cdot 2$ & 4915 & $9 \cdot 8$ & $8 \cdot 8,10 \cdot 8$ & 3223 & $8 \cdot 0$ & $6 \cdot 9,9 \cdot 3$ & 2322 & $4 \cdot 2$ & $3 \cdot 4,5 \cdot 2$ \\
\hline Amazon & 2741 & $8 \cdot 3$ & $7 \cdot 0,9 \cdot 8$ & 1989 & $4 \cdot 1$ & $3 \cdot 2,5 \cdot 1$ & 912 & $4 \cdot 4$ & $3 \cdot 4,5 \cdot 7$ & 957 & $3 \cdot 9$ & $2 \cdot 9,5 \cdot 2$ & 967 & $3 \cdot 4$ & $2 \cdot 5,4 \cdot 5$ \\
\hline
\end{tabular}

*Using the 2006 WHO Child Growth Standards ${ }^{(24)}$

tData are given for selected sociodemographic groups for which statistically significant differences in slopes were observed, e.g. urban $v$. rural, see Table 6. 
Table 6 Statistically significant differences in the regression $\beta$ coefficients (slopes of prevalence trends) between sociodemographic groups $^{*}$ for stunting (height-for-age $<-2$ SD below the median of the reference populationt) and overweight (weight-for-height $>+2$ SD above the median of the reference populationt) in children (aged 0-59 months). Peru Demographic and Health Surveys, 1991-2011

\begin{tabular}{|c|c|c|c|c|c|c|c|}
\hline & \multicolumn{2}{|c|}{ Group 1} & \multicolumn{2}{|c|}{ Group 2} & \multirow[b]{2}{*}{ Wald $(F)$} & \multirow[b]{2}{*}{$P \ddagger$} & \multirow[b]{2}{*}{ df } \\
\hline & $\beta$ & $95 \% \mathrm{Cl}$ & $\beta$ & $95 \% \mathrm{Cl}$ & & & \\
\hline \multicolumn{8}{|l|}{ Stunting } \\
\hline \multicolumn{8}{|l|}{ Residence } \\
\hline Urban§ v. Ruralll & -0.26 & $-0 \cdot 30,-0.22$ & $-0 \cdot 15$ & $-0 \cdot 18,-0 \cdot 11$ & $16 \cdot 8$ & $\leq 0.00$ & 1,5140 \\
\hline \multicolumn{8}{|l|}{ Geographic region } \\
\hline Coast $\S$ v. Andes $\|$ & -0.26 & $-0.31,-0.21$ & $-0 \cdot 13$ & $-0.17,-0.08$ & $15 \cdot 2$ & $\leq 0.00$ & 1, 4094 \\
\hline Andes $\S$ v. Amazon $\|$ & $-0 \cdot 13$ & $-0 \cdot 17,-0.08$ & $-0 \cdot 33$ & $-0.37,-0.28$ & $35 \cdot 6$ & $\leq 0.00$ & 1,2954 \\
\hline \multicolumn{8}{|l|}{ Maternal education } \\
\hline Complete secondary/higher§ $v$. Complete primary\| & $-0 \cdot 16$ & $-0 \cdot 21,-0 \cdot 12$ & -0.08 & $-0 \cdot 12,-0.03$ & $7 \cdot 9$ & $\leq 0.00$ & 1,4925 \\
\hline Complete primary $\S$. Incomplete primary $\|$ & -0.08 & $-0.12,-0.03$ & $-0 \cdot 14$ & $-0 \cdot 19,-0 \cdot 10$ & $5 \cdot 7$ & $\leq 0 \cdot 02$ & 1,3716 \\
\hline \multicolumn{8}{|l|}{ Overweight } \\
\hline \multicolumn{8}{|l|}{ Geographic region } \\
\hline Coast $\$$ v. Andes\| & 0.06 & $0 \cdot 02,0 \cdot 11$ & $-0 \cdot 10$ & $-0 \cdot 16,-0.05$ & $21 \cdot 0$ & $\leq 0.00$ & 1,4093 \\
\hline Coast $\S$ v. Amazon\| & 0.06 & $0 \cdot 02,0 \cdot 11$ & $-0 \cdot 26$ & $-0.35,-0.18$ & $42 \cdot 0$ & $\leq 0.00$ & 1,3352 \\
\hline Andes $\S v$. Amazon $\|$ & $-0 \cdot 10$ & $-0.16,-0.05$ & $-0 \cdot 26$ & $-0.35,-0 \cdot 18$ & $8 \cdot 6$ & $\leq 0 \cdot 00$ & 1,2953 \\
\hline
\end{tabular}

${ }^{*}$ Results are given only for significant differences.

tUsing the 2006 WHO Child Growth Standards ${ }^{(24)}$.

¥Statistical significance of the difference in regression coefficients between groups $(P<0.05)$.

$\S$ Group 1.

॥Group 2.

level of stunting of the WHO reference group. Knowing that all but a small fraction of children less than 59 months old avoid growth faltering when they develop in a healthy environment, the room for improvement in Peru remains large, and the present findings should serve as a call to renewed effort for child health in the country.

The national improvements in stunting might partly be accounted for by economic growth. Previous research has documented an association between economic development and health in Peru ${ }^{(38)}$. The Amazon region has documented increasing economic development in several domains from 2003-2007, almost on a level with national economic growth ${ }^{(39)}$. This could be one explanation for the decreasing stunting levels in this region. However, Acosta $^{(40)}$ argues that the improvements in child nutrition in the past decade are mainly due to the political efforts, particularly in the form of social welfare programmes, implemented to reduce poverty and poor health among vulnerable populations. Yamada et al. ${ }^{(41)}$, on the other hand, argue that such programmes have actually failed due to poor quality, design, targeting, low priority in times of recession and low fiscal priority compared with other countries in the region. Further, evaluations of specific programmes ${ }^{(2,42,43)}$ have failed to observe long-term reductions in child malnutrition. It is therefore difficult to determine the degree to which declining stunting trends observed in the present study are due to social welfare programmes. Besides social welfare programmes, national investments in health infrastructure may affect child malnutrition. Research on the effects of such investments on child stunting in Peru in recent decades shows a positive effect for urban children, with the effect being stronger for children in low-income groups compared with high-income groups, whereas no effect was found for rural children ${ }^{(44)}$. In spite of economic growth in recent decades ${ }^{(7,8)}$, social inequalities in nutritional status persist in Peru, disfavouring rural populations and people living in the Andean region ${ }^{(45)}$.

With regard to overweight, which is of mounting concern in the child health literature ${ }^{(4)}$, only a few subgroup analyses revealed a significant increase in overweight (male children in the coastal region and households in the richest wealth quintile). Conversely, significant declining trends were observed in many subgroups in the present study. Thus, the overall pattern in Peru is one of a stable trend in overweight. This is somewhat surprising, given the concern for an increase in child overweight in Latin America in recent years ${ }^{(46)}$. An increase in overweight would imply changes in the factors contributing to overweight (e.g. lifestyle changes affecting children) and based on our study findings we hypothesize that, overall, such changes have not been sufficient to result in a national upward trend of child overweight. However, this does not presume that no such changes have taken place. The upward overweight trends for some subgroups in the present study are consistent with other research in low- and middle-income countries, which observes that an increase in overweight is most often a phenomenon of the higher social classes ${ }^{(15,47,48)}$ and urban areas ${ }^{(47,48)}$. Increases in overweight have been attributed to lifestyle changes in specific population groups, for example in urban areas. Sedentary jobs and greater access to cheap, high-fat and processed foods can contribute to the increase in overweight observed in the present study as well as elsewhere ${ }^{(46-48)}$. Previous research observed that energy intake for children increased with household urbanization and increasing socio-economic status ${ }^{(49)}$ and that child overweight was positively associated with 
higher socio-economic status and a 'snacking dietary pattern' in urban children ${ }^{(50)}$. In rural areas, on the other hand, processed food is more expensive and the naturally grown foods are cheaper ${ }^{(46)}$, possibly resulting in a slower acceleration of overweight prevalence.

The considerations just mentioned point to the value of subgroup analyses, such as are presented in the current paper. The stratified analyses of stunting and overweight show that Peru, similar to its regional neighbours ${ }^{(21)}$, may be entering a nutritional transition that includes very young children. Although the findings in the present study do not indicate the typical 'from underweight to overweight' transition, the nutritional challenges may take on different forms depending on socio-economic status and type of residence, as is characteristic of the transition as observed in some other low- and middle-income countries $^{(15)}$.

As for all survey research, the DHS in Peru faces methodological challenges. As discussed by Pullum ${ }^{(51)}$, much of the DHS data are produced by self-reports of mothers about their own situation and experience and their child's health. Only anthropometric measurements are obtained independently and are clearly not affected by self-reporting bias. In addition, DHS analyses of seasonality effects indicate the possibility of seasonal variation in prevalence estimates for various health end points. It is a limitation of the DHS design that such seasonality effects cannot be eliminated or controlled for. There is some evidence that during the 3- to 6-month interval of fieldwork for the typical DHS survey, there is usually variation across months in the prevalence of some health measures. However, this is observed mainly for symptoms such as cough ${ }^{(51)}$. The extent to which such seasonality effects are evident for child growth measurement has not been addressed as far as we are aware. Regarding anthropometry, important sources of error include incorrect measurement of age, height/length and weight. As an example, DHS reports digit bias in the recording of child height, but concludes that digit bias is not likely to introduce an important level of error in the calculation of child growth variables.

A further limitation is the selection of a mother's youngest child as the index child, with all other children in the household excluded from study. The findings from the present study, therefore, cannot be generalized to the entire population of children aged 0-59 months.

The operational definitions of stunting and overweight deserve critical consideration. The standards used to determine stunting and overweight in the present study are those established by the WHO in $2006^{(24)}$ based on the results of the Multicentre Growth Reference Study. The intention of the WHO was to establish a new international standard, replacing the previous standard in which the reference population was restricted to children in the USA ${ }^{(52)}$. Because of this shift, the proportions of children who are classified as stunted and as overweight are higher under the new WHO standard than under the previous standard. The problem of two standards has been exacerbated in the literature, with some newer studies using the old standard and others using the new standard. The decision in the present study to use the new standard, and not present comparative analyses using the old standard, obscures the differences in stunting and overweight that result from the change in growth standards dating from 2006.

Aside from these general considerations, from DHS survey to survey, in country to country, local conditions have affected data collection. As an example from Peru, in 1991-92, sixty-six districts of the non-metropolitan domain had to be excluded due to inadequate conditions for data collection. The excluded districts were mainly small rural villages and some areas experiencing social violence at the time ${ }^{(29)}$. Rural residence and violent conditions are related to higher food insecurity and poorer medical care ${ }^{(53)}$ that might result in increased stunting prevalence. Hence, a possible consequence of the area exclusion is conservative stunting estimates for the 1991-92 (or following) data point.

It is also important to note that prior to 2003, DHS surveys were discrete activities. The continuous survey methodology was introduced from 2003, without the breaks between surveys that characterized the previous survey rounds. To preserve distinct cycles between surveys and due to practical issues such as the timing of the collection of anthropometric data, the second-to-last round of data was restricted to that collected in 2007-08 and the last round of data - collected in 2011 - was the latest available in time for inclusion in the present analysis. As a result, the interval between survey rounds used in the present study ranged from 3 to 7 years.

The regression analyses used to study trends assume linearity. An examination of the point prevalence estimates of stunting and of overweight call this assumption into question for some subgroups. In preliminary analyses not reported here due to space limitations, $\chi^{2}$ tests for homogeneity revealed some prevalence patterns that departed from the assumption of homogeneity. The decision to use a linear test of trend and not a nonlinear test followed from concern that few data points were available, just five in all. Nevertheless, the overall pattern of results seems to reasonably support our main conclusions.

Notwithstanding these limitations, the present study provides unique data about the trends in child growth in Peru, using the current recommended growth standards and operational definitions of stunting and overweight, and providing estimates of malnutrition for important demographic subgroups. It provides evidence that stunting is declining in almost all subgroups examined. However, particular subgroups continue to suffer from elevated levels of stunting, while other subgroups may be transitioning to join the global pandemic of overweight. 


\section{Acknowledgements}

Sources of funding: This research received no specific grant from any funding agency in the public, commercial or notfor-profit sectors. Conflicts of interest: The authors declare no conflict of interest. Ethics: Ethical approval was not required. Authors' contributions: H.B.U. did preliminary and final statistical analyses and wrote the first draft of this paper. She interpreted the results and contributed to later drafts, including the present manuscript. M.B.M. conceived of the study. He interpreted the results and contributed to later drafts, including the present manuscript. M.V. interpreted the results and contributed to later drafts, including the present manuscript. All authors were involved in data interpretation and critical revisions of the paper. All three authors read and approved the final revised draft for publication. Acknowledgements: The Demographic and Health Surveys of Peru for 1991, 1996, 2000, 2007-08 and 2011 were collected in collaboration with the Peruvian National Institute for Statistics (INEI), Asociación Benéfica PRISMA, the United Nations Development Programme (UNDP), UNICEF, the United Nations Population Fund (UNFPA), the US Agency for International Development (USAID) and the Peruvian Ministry of Economy and Finance. The authors would like to acknowledge the contributions made by collaborating partners, staff, field workers and participants of the Peru DHS.

\section{References}

1. Varela-Silva MI, Dickinson F, Wilson H et al. (2012) The nutritional dual-burden in developing countries - how is it assessed and what are the health implications? Coll Antropol 36, 39-45.

2. Stifel D \& Alderman H (2003) The 'Glass of Milk' subsidy program and malnutrition in Peru. World Bank Econ Rev 20, 421-428.

3. Albala C, Vio F, Kain J et al. (2001) Nutrition transition in Latin America: the case of Chile. Nutr Rev 59, 170-176.

4. Poskitt EME (2009) Countries in transition: underweight to obesity non-stop? Ann Trop Paediatr 29, 1-11.

5. Jehn M \& Brewis A (2009) Paradoxical malnutrition in mother-child pairs: untangling the phenomenon of overand under-nutrition in underdeveloped economies. Econ Hum Biol 7, 28-35.

6. Duran P, Caballero B \& de Onis M (2006) The association between stunting and overweight in Latin American and Caribbean preschool children. Food Nutr Bull 27, 300-305.

7. Economic Commission for Latin America and the Caribbean (2011) Preliminary Overview of the Economies of Latin America and the Caribbean 2011. Santiago: Economic Development Division, ECLAC.

8. United Nations Statistics Division (2012) UN Data: Peru country profile. http://data.un.org/CountryProfile.aspx? crName $=$ PERU $($ accessed December 2012).

9. Grantham McGregor S, Cheung YB, Cueto S et al. (2007) Developmental potential in the first 5 years for children in developing countries. Lancet 369, 60-70.

10. Black RE, Allen LH, Bhutta ZA et al. (2008) Maternal and child undernutrition: global and regional exposures and health consequences. Lancet 371, 243-260.
11. Victora CG, Adair L, Fall C et al. (2008) Maternal and child undernutrition: consequences for adult health and human capital. Lancet 26, 340-357.

12. Kelishadi R (2007) Childhood overweight, obesity, and the metabolic syndrome in developing countries. Epidemiol Rev 29, 62-76.

13. Daniels SR (2006) The consequences of childhood overweight and obesity. Future Child 16, 47-67.

14. Baird J, Fisher D, Lucas P et al. (2005) Being big or growing fast: systematic review of size and growth in infancy and later obesity. BMJ 331, 929.

15. El Taguri A, Besmar F, Abdel Monem A et al. (2009) Stunting is a major risk factor for overweight: results from national surveys of 5 Arab countries. East Mediterr Health J 15, 549-562.

16. Popkin B, Richards M \& Montiero C (1996) Stunting is associated with overweight in children of four nations that are undergoing the nutrition transition. J Nutr 126, 3009-3016.

17. Garrett JL \& Ruel MT (2008) Stunted child-overweight mother pairs: prevalence and association with economic development and urbanization. Food Nutr Bull 26, 209-221.

18. O'Donnel O, Van Doorslaer E, Wagstaff A et al. (2008) Analyzing Health Equity Using Household Survey Data. $A$ Guide to Techniques and Their Implementation. Washington, DC: The World Bank.

19. Amugsi DA, Mittelmark MB \& Lartey A (2013) An analysis of socio-demographic patterns in child malnutrition trends using Ghana demographic and health survey data in the period 1993-2008. BMC Public Health 13, 960.

20. Fernald LC \& Neufeld LM (2007) Overweight with concurrent stunting in very young children from rural Mexico: prevalence and associated factors. Eur J Clin Nutr 61, 623-632.

21. Rivera JA, Barquera S, González-Cossío T et al. (2004) Nutrition transition in Mexico and other Latin American countries. Nutr Rev 62, 149-157.

22. Mamabolo R, Alberts M, Steyn NP et al. (2005) Prevalence and determinants of stunting and overweight in 3-year-old black South African children residing in the Central Region of Limpopo Province, South Africa. Public Health Nutr $\mathbf{8}$, 501-508

23. Martorell R, Khan LK, Hughes ML et al. (2000) Overweight and obesity in preschool children from developing countries. Int J Obes Relat Metab Disord 24, 959-967.

24. World Health Organization (2006) WHO Child Growth Standards: Length/Height for Age, Weight for Age, Weight for Length, Weight for Height and Body Mass Index for Age: Methods and Development. Geneva: WHO.

25. Nichols EK, Nichols JS, Selwyn BJ et al. (2012) Implications of the WHO Child Growth Standards in rural Honduras. Public Health Nutr 15, 1015-1022.

26. De Onis M, Garza C, Onyango AW et al. (2007) Comparison of the WHO Child Growth Standards and the CDC 2000 Growth Charts. J Nutr 137, 144-148.

27. MEASURE DHS/ICF International (2012) Demographic and Health Surveys, DHS Methodology. http://www.measuredhs. $\mathrm{com} /$ What-We-Do/Survey-Types/DHS-Methodology.cfm (accessed August 2012).

28. MEASURE DHS/ICF International (2012) Demographic and Health Survey Sampling and Household Listing Manual. Calverton, MD: MEASURE DHS/ICF International.

29. Padilla A, Ochoa LH \& Marckwardt AM (1992) PERÚ Encuesta Demográfica y de Salud Familiar 1991/1992. Lima and Calverton, MD: Instituto Nacional de Estadística e Informática and ORC Macro.

30. Reyes J \& Ochoa LH (1997) PERÚ Encuesta Demográfica y de Salud Familiar 1996. Lima: Instituto Nacional de Estadística e Informática. 
31. Reyes J \& Ochoa LH (2001) PERÚ Encuesta Demográfica y de Salud Familiar 2000. Lima and Calverton, MD: Instituto Nacional de Estadística e Informática and Macro International/ Measure DHS+.

32. Instituto Nacional de Estadística e Informática (2009) PERÚ Encuesta Demográfica y de Salud Familiar 2007-2008 Informe Principal. Lima: INEI.

33. Instituto Nacional de Estadística e Informática (2012) Perú Encuesta Demográfica y de Salud Familiar 2011. Lima: INEI.

34. World Health Organization (2006) A Macro/Programme for Calculating the Z-scores and Prevalences for DHS Individual Flat Files. Geneva: WHO Department of Nutrition for Health and Development.

35. World Health Organization (2010) WHO Anthro for Personal Computers, version 3.1, 2010: Software for Assessing Growth and Development of the World's Children. Geneva: WHO.

36. ICF International (2012) Measure DHS Biomarker Field Manual. Calverton, MD: ICF International.

37. Rutstein O \& Johnson K (2004) The DHS Wealth Index. Calverton, MD: ORC Macro.

38. Pritchett L \& Summers LH (1993) Wealthier is Healthier. Policy Research Working Paper no. WPS 1150. Washington, DC: World Bank.

39. Yamada G (2010) Growth, Employment, and Internal Migration. Peru, 2003-2007. Paper no. 22067. Lima: Universidad del Pacífico.

40. Acosta AM (2011) Analysing Success in the Fight against Malnutrition in Peru. IDS Paper no. 367. Brighton: Insitute of Development Studies.

41. Yamada G \& Castro JF (2012) Poverty, Inequality, and Social Policies in Peru: As Poor As it Gets. The Peruvian Growth Puzzle. Boston, MA and Lima: Center for International Development, Harvard University and Centro de Investigación de la Universidad del Pacífico.

42. Gajate GG \& Inurritegui MM (2002) El impacto de los programas alimentarios sobre el nivel de nutrición infantil: una aproximación a partir de la metodología del 'Propensity Score Matching'. Lima: GRADE.

43. Perova E \& Vakis R (2009) Welfare Impacts of the 'Juntos' Program in Peru: Evidence from a Non-Experimental Evaluation. Washington, DC: World Bank.

44. Valdivia M (2004) Poverty, health infrastructure and the nutrition of Peruvian children. Econ Hum Biol 2, 489-510.

45. Urke H, Bull T \& Mittelmark M (2011) Socioeconomic status and chronic child malnutrition: wealth and maternal education matter more in the Peruvian Andes than nationally. Nutr Res 31, 741-747.

46. Uauy R, Albala C \& Kain J (2001) Obesity trends in Latin America: transiting from under- to overweight. J Nutr 131, issue 3, 893S-899S.

47. Kain J, Vio F \& Albala C (2003) Obesity trends and determinant factors in Latin America. Cad Saude Publica 19, 77-86.

48. Popkin B, Adair L \& Ng SW (2012) Global nutrition transition and the pandemic of obesity in developing countries. Nutr Rev 70, 3-21.

49. Kelles A \& Adair L (2009) Offspring consume a more obesogenic diet than mothers in response to changing socioeconomic status and urbanization in Cebu, Philippines. Int J Behav Nutr Phys Act 6, 47.

50. McDonald C, Baylin A, Arsenault J et al. (2009) Overweight is more prevalent than stunting and is associated with socioeconomic status, maternal obesity, and a snacking dietary pattern in school children from Bogotá, Colombia. J Nutr 139, 370-376.

51. Pullum T (2008) An Assessment of the Quality of Data on Health and Nutrition in the DHS Surveys, 1993-2003. Methodological Reports no. 6. Calverton, MD: Macro International Inc.

52. Waterlow JC, Buzina R, Keller W et al. (1977) The presentation and use of height and weight data for comparing the nutritional status of groups of children under the age of 10 years. Bull World Health Organ 55, 489-498.

53. Krug EG, Dahlberg LL, Mercy JA et al. (2002) World Report on Violence and Health. Geneva: WHO. 